conditions. Immunosuppression frequently occurs in chronic lymphocytic leukaemia and coeliac disease, and both of these conditions are associated with an increased incidence of malignant disease.

Choriocarcinoma and Burkitt's lymphoma are unique among malignant tumours in that high rates of cure can be obtained with chemotherapy. Evidence for the existence of foreign antigens on the tumour membrane is best in these cancers, and it is tempting to think that immune mechanisms may be responsible for the good prognosis. On the other hand, similar immune responses against tumour-associated antigens have been clearly shown in conditions which do not carry such a good prognosis, such as nasopharyngeal carcinoma, osteogenic sarcoma, malignant melanoma, neuroblastoma, and carcinoma of the colon and bladder. In melanoma, for example, antibody to the tumour has been found in the sera of patients with localized disease, whereas the antibody disappears when the tumour becomes disseminated. Possibly this antibody may have a role in preventing the spread of metastases in the blood stream, but, disappointingly, immunization with the patient's own irradiated malignant melanoma cells did not affect the disease.

Even if the presence and strength of an immunological reaction to a tumour could be correlated closely with the prognosis, this would not necessarily mean that the improved outlook was due to the immune response. The immune response may merely be related to the amount of degeneration of the tumour, or other factors more directly concerned with a favourable outlook. In view of these difficulties, the evidence that immunological reactions are of value in host resistance must depend on the results of treatment. The regional lymph nodes play an extremely important part in the rejection of a homograft and the same is true in the rejection of antigenic animal tumours. Thus it would seem reasonable to retain the normal regional lymphoid tissue in patients with cancer provided the tumour has not invaded them. The difficulty lies in knowing whether the lymph nodes are involved or not. In stage I carcinoma of the breast Professor Crile has shown that the rate of survival is $15^{\circ}$ ", higher if the nodes are not removed or irradiated. On the other hand, in other forms of tumour removal or irradiation of apparently uninvolved local lymph nodes may improve survival. Hence it is impossible to generalize.

Unfortunately, there are many difficulties in the path of successful immunotherapy. Even the most antigenic tumours in animals induce only very weak immunity, so that only a few tumour cells can be rejected and larger tumours grow well even in the presence of active tumour immunity. A corollary of this in man is that, if immunotherapy is to have a reasonable chance, the number of malignant cells in a patient must be reduced to a minimum by conventional therapy before immunotherapy is given. Even so, treatment should be given with caution. In animals some forms of immunotherapy have had no effect on the tumour at all, while occasionally treatment with antibody has led to tumour growth actually being enhanced.

With the exception of one study by Professor Georges Mathé, there is no evidence so far that immunological procedures are useful in controlling disseminated malignant disease in man. This study concerned 30 patients with acute lymphoblastic leukaemia in remission after two years' intensive chemotherapy. Ten were given no further treatment and relapsed within 130 days. Twenty patients were given B.C.G. or irradiated leukaemic cells, or both. Seven patients are still in remission more than two years after stopping chemotherapy, five for more than three years, and two for more than four years. These results arouse hope that future methods of stimulating host resistance to human cancer may be found which will be applicable to a wider variety of human tumours.

\section{Are Antidiabetic Drugs Dangerous?}

On 6 November the American Food and Drug Administration issued a warning to doctors about the possible dangers of oral antidiabetic drugs. It recommended that these should be given only to patients who cannot be controlled by diet or weight loss and for whom the addition of insulin is impracticable or unacceptable. The American Diabetes Association and the Council on Drugs of the American Medical Association have now added their support to this view.

The evidence for this recommendation came from the results of a co-operative study (the University Group Diabetes Program), which was started in 1961 in six university centres, later expanded to 12. The final results have not yet been published but are due to appear as a supplement to the November issue of Diabetes. Nevertheless, they are unlikely to differ substantially from those in the draft report by the University Group Diabetes Program in September of this year, which gives full details of the trial and its results. ${ }^{1}$

This co-operative study was designed to answer the question: Does treatment "postpone the appearance and delay the progression of the vascular complications associated with diabetes?" Eight hundred newly or recently diagnosed diabetics who were not dependent on insulin were recruited within five years, and they have been studied for up to eight and threequarter years, more than three-quarters for over five years. The patients were randomly assigned to one of four treatment groups-namely, placebo (lactose), tolbutamide $1.5 \mathrm{~g}$. daily, insulin in a fixed dose (12-16 units according to body surface), or insulin in a variable dose according to need. In addition all the patients were given advice on a suitable diet to follow.

The main finding is that there was a significantly higher death rate from cardiovascular disease in the tolbutamide group than in the placebo group. The numbers of deaths from cardiovascular disease (with percentages in parentheses) were as follows in the four groups: placebo $10(4 \cdot 9)$, tolbutamide $26(12 \cdot 7)$, insulin fixed dose $13(6 \cdot 2)$, and insulin variable dose $12(5 \cdot 9)$. The results for patients in the two groups on insulin are considered to be inconclusive. The difference between the number of deaths from all causes in the placebo group (21) and the tolbutamide group (30) was smaller than the difference between the numbers of deaths from cardiovascular disease, and was not significant by conventional criteria.

These findings are striking and at first sight might suggest that tolbutamide therapy has dangers. Nevertheless, the case against tolbutamide is far from overwhelming, and several criticisms must be answered before the programme's conclusion can be accepted. In particular, the trial itself can be criticized on several grounds. Firstly it was conducted in 12 centres, each contributing 44-94 cases. Though the object of using several centres was to collect sufficient patients, the contribution of the individual centres was in fact relatively

University Group Diabetes Program. A Study of the Effects of Hypoglycaemic Agents on Vascular Complications in Patients with Adult-Onset Diabetes. New York, 1970

2 Keen, H., and Jarrett, R. H., in Atherosclerosis: Proceedings of the Second Symposium, ed. R. J. Jones, pp. 435-443. New York, Springer-Verlag,

${ }^{3}$ Passikivi, J., Acta Medica Scandinavica, 1970, Suppl. No. 507.

4 Newsweek, 2 November 1970. 
small, and it took about 70 clinic-years to assemble the 800 patients studied. Moreover, the results from all the centres are not consistent. While two centres, Cincinatti and Minneapolis, contributed less than a quarter of all the patients they provided no fewer than half the deaths in the placebo and tolbutamide groups. The accuracy of some of the causes of death given must be in doubt, since necropsies were done in only one-third of the cases and in different proportions in the two groups (half in the tolbutamide group, one-quarter in the placebo group). More of the deaths in the tolbutamide group occurred in hospital $(70 \%)$ than in the placebo group (52\%). On the other hand, of 205 middle-aged diabetics in the placebo group observed for up to eight and three-quarter years not one is recorded as having died from myocardial infarction.

Another unusual feature of this study, in which as many as $72 \%$ of the patients were women, was that the excess mortality from cardiovascular disease among the tolbutamide group was almost confined to women. Among the men there were 7 deaths in the placebo group as against 11 in the tolbutamide group, compared with 3 and 15, respectively, in the women. Again, of all the patients in the study half were non-white (a much higher proportion than in the general population); and the excess of female cardiovascular deaths was mainly among white women. Hence in so far as this study suggests an increased risk of cardiovascular death among diabetics treated with tolbutamide, this risk is mainly for white women.

These results are at variance with those reported by $\mathrm{H}$. Keen and R. J. Jarrett in Great Britain ${ }^{2}$ and by J. Paasikivi in Sweden. ${ }^{3}$ Neither of these studies shows an increased risk of cardiovascular disease in the groups of patients treated with tolbutamide. In fact, they suggest the reverse.

If the results are in some ways open to question, so too is the way they have been handled. It is desirable that the results of drug trials, as of other medical research, should be assessed in a cool and critical fashion. This becomes difficult when they reach the lay press before doctors as a whole have had the chance to consider them, as has happened in the present case. ${ }^{4}$ Why have the conclusions drawn from this report been so urgent, certain, and final? The tolbutamide study has now been shut down. The trial is in many ways thorough and excellent, but several criticisms can be made and the F.D.A. itself admits that it contains "a number of limitations." In June the American Diabetes Association issued a statement saying that the evidence did "not appear to warrant abandoning the present accepted methods of treatment of diabetes-diet, diet with oral agents, or diet and insulin as indicated." Yet now, with no new evidence available, the Ar.terican Diabetes Association has associated itself with the F.D.A. view that oral drugs should be used only when diet alone is ineffective and insulin is impracticable or unacceptable. This advice is the more surprising since the co-operative study showed that less than half the patients on a variable dose of insulin actually adhered strictly to their treatment. Moreover, insulin treatment itself has drawbacks, including hypoglycaemia and weight gain, with the possible risks resulting from them.

Since the co-operative study has not yet been published in its final form the medical profession in general, whether in the U.S.A. or elsewhere, has had no opportunity to evaluate it. That it needs unusually careful scrutiny is certain, for some aspects of it are open to question. In Britain it would be wise to defer judgement on what action to take uncil the report has been published and studied rather than to follow blindly the F.D.A.'s lead. The calm attitude throughout of the British Diabetic Association, which has also advised caution, is to be welcomed.

\section{The Immunoglobulins}

When A. Tiselius ${ }^{1}$ in 1937 first separated the plasma proteins by their different mobilities in an electric field, he distinguished three varieties of globulin designated $\alpha, \beta$, and $\gamma$ in decreasing order of migration. It soon became apparent that the antibody property of an immune serum - that is, its capacity to react specifically in a detectable fashion with some foreign substance-resided in the $\gamma$ globulin fraction, and the term "immunoglobulin" is now applied to any constituent of this fraction that can be shown to possess either specific antibody properties or the structure now known to be characteristic of them.

By a combination of physical, chemical, and immunological methods the human immunoglobulins have been divided into five classes designated $\mathrm{G}, \mathrm{A}, \mathrm{M}, \mathrm{D}$, and $\mathrm{E}$. In normal human serum $\mathrm{G}$ is the most abundant and $\mathrm{E}$ the least. All five classes are constructed on the same basic plan-namely, four peptide chains, two short and two long, the former each composed of about 214 amino-acids, the latter of about twice that number. They are now generally referred to as the light (L) and heavy $(\mathrm{H})$ chains with respective molecular weights of about 25,000 and 50,000 , the complete molecule thus possessing a molecule weight of 150,000 . Whereas most of the molecules of immunoglobulin $\mathrm{G}$ (IgG) exist in this form, the IgM molecule is made up of five such units covalently linked to each other by sulphur bonds between cysteine residues. By careful chemical reduction of these bonds the IgM molecule can be dissociated into its constituent units of about 150,000 molecular weight. IgD and $\operatorname{IgE}$ probably exist mainly in the monomer form (single units) like IgG, but IgA shows a considerable tendency to aggregate into dimers and larger polymers.

The difference between the five classes of immunoglobulin lies exclusively in the heavy chains. The light chains, of which two forms exist, are common to all the immunoglobulins, but each class, G, A, M, D, and E, has its own characteristic heavy chain designated $\gamma, \alpha, \mu, \delta$, and $\varepsilon$ respectively. These, being immunologically distinct, can be readily detected and differentiated by specific antibodies.

The mutual relationship of the four constituent chains in the basic immunoglobulin molecule has been largely established by the study of the fragments released by proteolytic enzymes. Thus R. R. Porter ${ }^{2}$ showed that rabbit immunoglobulin digested with papain yielded two distinct types of fragment. Only one of them retained the antibody specificity of the parent molecule and was therefore labelled Fab (i.e., antibody containing fragment), while the other, the Fc fraction, had no such biological activity but could be readily crystallized, indicating a degree of homogeneity not present in the Fab fraction. The Fab fragments, though retaining antibody specificity, were incapable of precipitating antigen, because each fragment retained only one of the two combining sites of the parent molecule. But A. Nisonoff and colleagues ${ }^{3}$ showed that if pepsin was used in place of papain the homologous fragment, now termed $F\left(a b^{\prime}\right)_{2}$, still retained the capacity of immunologically specific precipitation and was thus still bivalent. The results of these and similar experiments led to the Porter diagram ${ }^{4}$ of immunoglobulin structure, which also shows the makeup of the various fragments of proteolytic digestion (see Fig.).

Although the $\mathrm{Fc}$ portion of the $\mathrm{H}$ chains plays no part in the immunological specificity of the various immunoglobulins, it is of considerable biological significance, because the properties of complement fixation, ability to pass the 